\title{
Comparison of patients with multivessel disease treated at centers with and without on-site cardiac surgery
}

Eilon Ram, MD, ${ }^{\mathrm{a}}$ Ilan Goldenberg, MD, ${ }^{\mathrm{b}}$ Yigal Kassif, MD, ${ }^{\mathrm{a}}$ Amit Segev, MD, ${ }^{\mathrm{b}}$ Jakob Lavee, MD,

Nir Shlomo, MSc, ${ }^{\mathrm{b}}$ and Ehud Raanani, MD

\section{ABSTRACT}

Background: The regional needs and consolidation of cardiac surgery services (CSS) result in an increased number of stand-alone interventional cardiology units. We aimed to explore the impact of a heart team on the decision making and outcomes of patients with multivessel coronary artery disease referred for coronary revascularization in stand-alone interventional cardiology units.

Methods: This prospective study included 1063 consecutive patients with multivessel disease enrolled between January and April 2013 from all 22 hospitals in Israel that perform coronary angiography and percutaneous coronary intervention (PCI), with or without on-site CSS.

Results: Of the 1063 patients, 487 (46\%) underwent coronary artery bypass grafting (CABG) and 576 (54\%) underwent PCI. A higher proportion of patients underwent PCI in hospitals without on-site CSS compared with those with on-site CSS $(65 \%$ vs $46 \% ; P<.001)$. Furthermore, patients referred to CABG from hospitals without on-site CSS had a significantly higher mean SYNTAX score compared with those who underwent CABG in centers with on-site CSS (29 vs 26; $P=.018$ ). Multivariate logistic regression analysis consistently showed that the absence of on-site cardiac surgery and a heart team was independently associated with a 2.5-fold increased likelihood for predicting the referral of PCI rather than $\mathrm{CABG}$ (odds ratio, 2.54; 95\% confidence interval, 1.8-3.6).

Conclusions: Patients with multivessel coronary artery disease treated in centers without on-site cardiac surgery services receive a lower rate of appropriate guideline-based intervention with CABG. These findings suggest that a heart team approach should be mandatory even in centers with stand-alone interventional cardiology units. (J Thorac Cardiovasc Surg 2018;155:865-73)

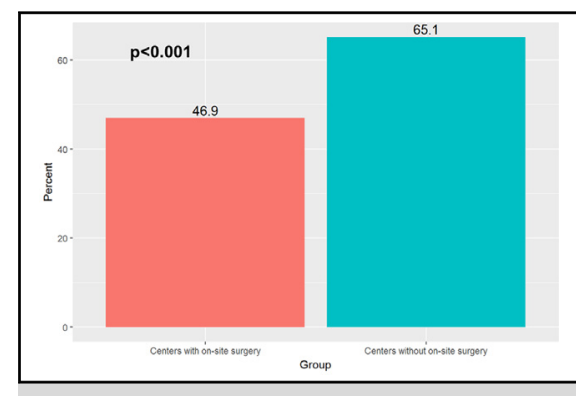

Bar graph of percentage of patients who underwent $\mathrm{PCl}$ by type of center.

\section{Central Message}

Patients with multivessel coronary artery disease treated in centers without on-site cardiac surgery services receive a lower rate of appropriate guideline-based intervention with CABG.

\section{Perspective}

Lack of a heart team raises the potential for significant bias in referral patterns for coronary revascularization in stand-alone interventional cardiology units. Our findings suggest that a heart team approach should be mandatory even in centers with stand-alone interventional cardiology units, thus positively influencing the quality of cardiovascular care.

See Editorial Commentary page 874.
Ischemic heart disease is the leading cause of death globally that accounts for about 7.4 million mortalities worldwide annually as published by the World Health Organization.

\footnotetext{
From the Departments of ${ }^{\mathrm{a} C a r d i a c}$ Surgery and ${ }^{\mathrm{b}}$ Cardiology, Leviev Cardiothoracic and Vascular Center, Sheba Medical Center, affiliated with the Sackler School of Medicine, Tel Aviv University, Tel Aviv, Israel.

Read at the 97th Annual Meeting of The American Association for Thoracic Surgery, Boston, Massachusetts, April 29-May 3, 2017.

Received for publication May 24, 2017; revisions received Aug 31, 2017; accepted for publication Sept 26, 2017.

Corresponding author: Ehud Raanani, MD, Department of Cardiac Surgery, Sheba Medical Center, Tel Hashomer 52621, Israel (E-mail: ehud.raanani@sheba. health.gov.il).

$0022-5223 / \$ 36.00$

Copyright $(2017$ by The American Association for Thoracic Surgery

https://doi.org/10.1016/j.jtcvs.2017.09.144
}

Coronary artery bypass grafting (CABG) and percutaneous coronary intervention (PCI) are the options for revascularization in patients suffering from coronary artery disease (CAD). The choice of the most appropriate modality remains controversial in some patient groups. ${ }^{2-5}$ Hence, it

Scanning this QR code will
take you to a supplemental
video, figure, and tables. To
view the AATS Annual
Meeting Webcast, see the
URL next to the webcast
thumbnail.




\section{Abbreviations and Acronyms}

$\mathrm{CABG}=$ coronary artery bypass grafting

$\mathrm{CAD}=$ coronary artery disease

CSS = cardiac surgery services

LAD $=$ left anterior descending artery

PCI = percutaneous coronary intervention

is recognized that in many cases an individualized approach should be taken in order to determine the best interventional approach for each specific patient.

As a result, a heart team comprising a cardiac surgeon, an interventional cardiologist and a general cardiologist has been accepted and recommended by the current guidelines, in order to decide between CABG and/or PCI. ${ }^{6-8}$ The heart team should review the patient's medical condition and coronary anatomy, determine that $\mathrm{CABG}$ and/or PCI are technically feasible and reasonable, put into context the clinical condition of the patient and discuss revascularization options with the patient before a treatment strategy is selected. By adopting this approach, the heart team is able to minimize specialty bias and prevent self-referral from interfering with optimal patient care.

In an era when an increasing number of large cardiac surgery departments continue to grow, there is a trend towards closing smaller departments and consolidating cardiac surgery services (CSS) based on regional considerations, which in turn results in an increased number of stand-alone interventional cardiology units. The lack of on-site CSS may result in less patient-oriented heart teams and potentially inherent bias towards higher rates of PCI. We aimed to explore the impact of the lack of a heart team in stand-alone interventional cardiology units on decision making regarding patients with multi-vessel coronary disease enrolled in a prospective real-life national registry. The primary focus of the study was to identify the differences in referral patterns and management of revascularization strategies (ie, CABG vs PCI) between centers with and without available on-site CSS and a heart team in patients with multivessel CAD (Video 1).

\section{METHODS \\ Study Design}

The present study population was taken from the prospective Multivessel Coronary Artery Disease (MULTICAD) Israeli Registry, which included all 22 hospitals in Israel that perform coronary angiography and PCI, with or without on-site CSS. The aim of the MULTICAD registry is to provide contemporary data on real-life management strategies of patients with multivessel CAD in Israel, provide information regarding the clinical course following $\mathrm{CABG}$ and multivessel PCI, identify risk factors for adverse events following revascularization, and compare the 2 revascularization approaches in risk subgroups defined by coronary anatomy and clinical factors. Among the 22 hospitals in Israel that perform coronary angiography and PCI, 9 have an on-site cardiac surgery

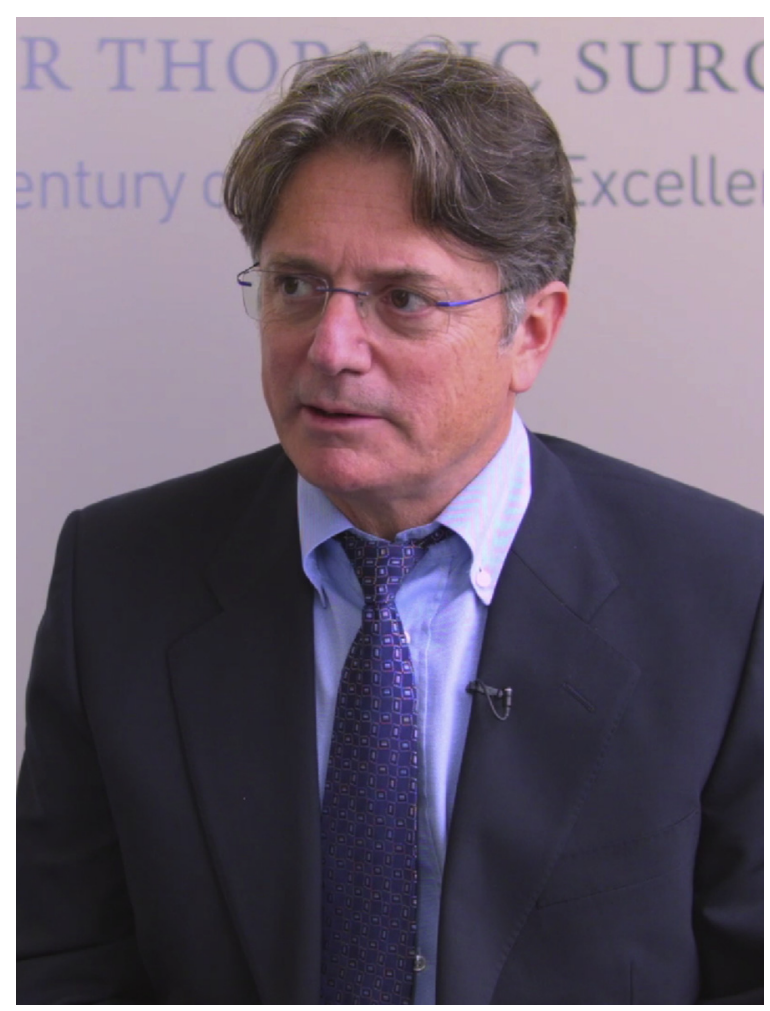

VIDEO 1. An interview with Professor Ehud Raanani, explaining the importance and relevance of this study. Video available at: http://www. jtcvsonline.org/article/S0022-5223(17)32477-7/fulltext.

department or unit, and 13 do not. A formal heart team was coordinated according to the discretion of the treating physician, rather than relying on uniform decision making.

From January through April 2013, 1112 consecutive patients were hospitalized with multi-vessel CAD. Of them, 1063 patients met the eligibility criteria, which included all patients age $\geq 18$ years with multivessel CAD, defined as 2- or 3-vessel CAD involving the proximal or mid-left anterior descending artery (LAD) and any unprotected left main artery with $>50 \%$ narrowing with or without additional CAD. These patients were enrolled and prospectively followed over a 3-year period. Exclusion criteria included patients who had undergone previous CABG and/or were treated with primary PCI at the current admission, those with moderate or severe valvular disease, and pregnant or nursing women. A total of 49 patients were excluded from the study: 11 treated with a combined revascularization strategy with CABG and PCI, and 38 treated conservatively (Figure 1). All patients included in this study were treated by either $\mathrm{CABG}$ or PCI; referral to one revascularization strategy was synonymous with eventually undergoing the revascularization by the same strategy. The choice of revascularization strategy was at the discretion of the treating team. The study was approved by the Institutional Review Board of each participating center, and all patients provided informed consent.

\section{Data Collection and Follow-up}

All hospital data were collected and pooled into a designated database using standardized definitions. Data included demographic parameters, medical history, chronic and periprocedural medical treatment, echocardiography measurements, procedural information, and outcome measures. All coronary catheterization compact discs were evaluated, and the coronary findings were adjudicated at a core laboratory 


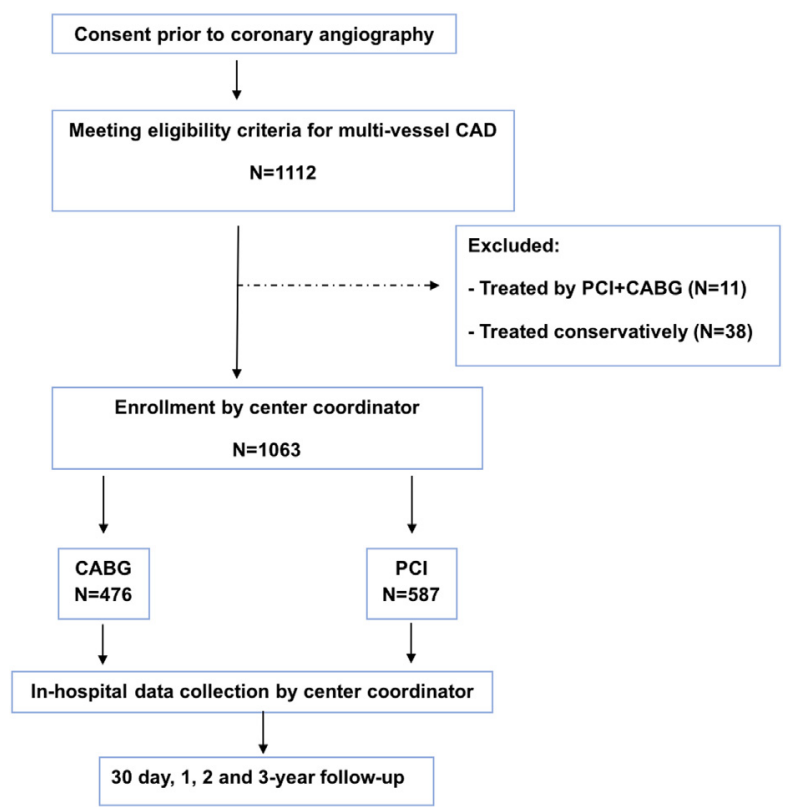

FIGURE 1. Flow chart summary from consent and eligibility, through a 3-year follow-up. $C A D$, Coronary artery disease; $P C I$, percutaneous coronary intervention; $C A B G$, coronary artery bypass graft.

(Rambam Medical Center). All patients were prospectively followed up for clinical events at 30 days (Table E1) and for mortality at 36 months. Mortality was ascertained from the Israeli Ministry of Interior Population Register through April 2016.

\section{Statistical Analysis}

Data are presented as mean (standard deviation) when normally distributed or as median when not normally distributed. Continuous variables were tested with the Kolmogorov-Smirnov test for normal distribution. Categorical variables are given as frequencies and percentages. The $\chi^{2}$ test was used for comparison of categorical variables between patients treated in centers with and without on-site CSS, and Student's $t$ test was performed for comparison of continuous variables between the 2 groups. Multivariate logistic regression analysis was used to identify factors associated with referral to PCI. Candidate factors are presented in Tables 1 and 2. In a secondary analysis, consistency of the findings in the primary logistic regression model was assessed using a logistic regression model with a mixed-effects analysis to adjust for differences between centers and within-center variation using the lme4 package.

Survival analyses were done using the Kaplan-Meier method, and statistical differences by the presence of on-site CSS were tested using the log-rank test. In addition, subgroup analysis was based on the SYNTAX score (an angiographic grading tool to determine the complexity of the CAD), defined as follows: low SYNTAX score, $\leq 22$; intermediate SYNTAX score, 23-32; high SYNTAX score, $>32$.

Multivariate Cox proportional hazard modeling was used to identify factors associated with mortality risk at 3 years, with candidate factors listed in Tables 1 and 2. A stepwise method was used to identify independent predictors of mortality. Violation of the proportional hazards assumption was tested using the Cox.zph function, which tests proportionality of all the model predictors. In a secondary analysis, consistency of the findings in the primary multivariate Cox model was assessed using propensity score matching, with further adjustment for the Euroscore as an additional covariate among patients with available data on all Euroscore parameters $(n=604 ; 57 \%)$, and a Cox proportional hazard model with mixed effect analysis to adjust for differences between centers and within-center variation using the coxme package.
TABLE 1. Baseline patient characteristics

\begin{tabular}{|c|c|c|c|}
\hline Characteristic & $\begin{array}{c}\text { Centers } \\
\text { without CSS } \\
(n=487)\end{array}$ & $\begin{array}{c}\text { Centers } \\
\text { with CSS } \\
(n=576)\end{array}$ & $\begin{array}{c}P \\
\text { value }\end{array}$ \\
\hline Age, y, mean (SD) & $66(11)$ & $65(11)$ & .36 \\
\hline Male sex, n (\%) & $373(77)$ & $474(82)$ & .03 \\
\hline Current smoker, n (\%) & $135(28)$ & $151(26)$ & .63 \\
\hline Hyperlipidemia, n (\%) & $351(73)$ & $428(76)$ & .29 \\
\hline Diabetes mellitus, n (\%) & $227(47)$ & $248(43)$ & .33 \\
\hline Renal impairment, n (\%) & $54(11)$ & $74(13)$ & .42 \\
\hline COPD, n $(\%)$ & $37(8)$ & $36(6)$ & .47 \\
\hline Previous IHD, n (\%) & $131(27)$ & $168(30)$ & .34 \\
\hline Previous PCI, n (\%) & $181(37)$ & $184(32)$ & .10 \\
\hline History of CVA/TIA, n (\%) & $47(10)$ & $51(9)$ & .76 \\
\hline NYHA score III-IV, n (\%) & $10(17)$ & $11(31)$ & .21 \\
\hline \multicolumn{4}{|l|}{ Indication for angiography } \\
\hline Stable angina, $\mathrm{n}(\%)$ & $134(27)$ & $138(24)$ & .21 \\
\hline ACS, n $(\%)$ & $312(64)$ & $355(62)$ & .45 \\
\hline CHF, n $(\%)$ & $13(3)$ & $14(2)$ & .96 \\
\hline Other, n $(\%)$ & $28(6)$ & $69(12)$ & .01 \\
\hline \multicolumn{4}{|l|}{ Medications on admission } \\
\hline Aspirin, $\mathrm{n}(\%)$ & $325(67)$ & $400(75)$ & .01 \\
\hline Beta blockers, n (\%) & $246(51)$ & $294(52)$ & .74 \\
\hline ACE inhibitors, $\mathrm{n}(\%)$ & $231(48)$ & $249(44)$ & .26 \\
\hline Statins $(\%)$ & $330(68)$ & $401(71)$ & .34 \\
\hline Antihyperglycemic, n (\%) & $150(31)$ & $142(30)$ & .82 \\
\hline Fusid, n (\%) & $58(12)$ & $46(10)$ & .46 \\
\hline Coumadin, n (\%) & $15(3)$ & $14(3)$ & 1.00 \\
\hline
\end{tabular}

$C S S$, Cardiac surgery services; $S D$, standard deviation; $C O P D$, chronic obstructive pulmonary disease; $I H D$, ischemic heart disease; $P C I$, percutaneous coronary intervention; CVA, cerebrovascular accident; TIA, transient ischemic attack; NYHA, New York Heart Association; ACS, acute coronary syndrome; CHF, congestive heart failure; $A C E$, angiotensin-converting enzyme.

Statistical significance was assumed when the null hypothesis could be rejected at $P<.05$. All $P$ values are results of 2 -sided tests. Statistical analyses were conducted using $\mathrm{R}$ version (R Foundation for Statistical Computing, Vienna, Austria). The investigators initiated the study, had full access to and analyzed all the data, and wrote the manuscript. All authors vouch for the data and analysis.

\section{RESULTS}

\section{Baseline Clinical Characteristics}

Among the 1063 patients included in the study, 576 $(54 \%)$ underwent coronary angiography in the 9 centers with on-site CSS, compared with $487(46 \%)$ in the 13 centers without on-site CSS. The mean age of the study patients was 65 years, and $20 \%$ were women. Baseline clinical characteristics of the study patients by the availability of on-site CSS are presented in Table 1. Notably, there were no statistically significant differences in most baseline clinical parameters between those who underwent coronary angiography in centers with on-site CSS and those who did so in centers without on-site CSS. 
TABLE 2. Angiographic characteristics

\begin{tabular}{|c|c|c|c|}
\hline Characteristic & $\begin{array}{c}\text { Centers } \\
\text { without CSS } \\
(n=487)\end{array}$ & $\begin{array}{l}\text { Centers } \\
\text { with CSS } \\
(\mathbf{n}=\mathbf{5 7 6})\end{array}$ & $\begin{array}{c}P \\
\text { value }\end{array}$ \\
\hline Left main, n (\%) & $113(23)$ & $139(24)$ & .78 \\
\hline Proximal LAD, n (\%) & $229(47)$ & $269(47)$ & .96 \\
\hline 2-vessel disease, n (\%) & $195(40)$ & $199(35)$ & .08 \\
\hline$\geq 3$ - vessel disease, $\mathrm{n}(\%)$ & $237(49)$ & $309(54)$ & .08 \\
\hline $\begin{array}{l}\text { SYNTAX score, n (\%) } \\
\text { High }(>32) \\
\text { Medium }(23-32) \\
\text { Low }(\leq 22)\end{array}$ & $\begin{array}{r}72(16) \\
114(26) \\
256(58)\end{array}$ & $\begin{array}{r}72(14) \\
158(31) \\
282(55)\end{array}$ & .196 \\
\hline SYNTAX score, mean (SD) & $22.13(10.36)$ & $22.13(9.98)$ & .995 \\
\hline
\end{tabular}

$C S S$, Cardiac surgery services; $L A D$, left anterior descending; $S D$, standard deviation.

These clinical parameters included age, renal impairment, diabetes mellitus, hypertension, chronic obstructive pulmonary disease, previous cardiovascular disease, and a previous cerebrovascular event. However, centers without on-site CSS had a somewhat higher number of women (24\% vs $18 \% ; P=.026)$, and a higher proportion of patients with previous PCI $(37.2 \%$ vs $32.2 \% ; P=.096)$. Among patients with available data on all Euroscore parameters $(\mathrm{n}=604 ; 57 \%)$, the mean Euroscore was 1.23 among patients hospitalized in centers with on-site CSS versus 1.40 among those hospitalized in centers without CSS $(P=.049)$.

Among all study patients, indications for the index coronary angiography were as follows: stable angina pectoris in $25 \%$, acute coronary syndrome in $63 \%$, and congestive heart failure symptoms in $3 \%$, without statistical significant differences between those enrolled in centers with on-site CSS and those in centers without on-site CSS (Table 1). In addition, baseline cardiovascular medical therapies were similar between the 2 groups, except for the use of aspirin therapy, which was higher in patients admitted to centers with on-site CSS.

\section{Baseline Angiographic Characteristics}

Among all study patients, the frequency of left main artery disease was $24 \%$, the frequency of proximal LAD disease was $47 \%$, and $51 \%$ had involvement of $\geq 3$ main epicardial arteries. The mean SYNTAX score was $22 \pm 10$.

A comparison of the angiographic characteristics of patients enrolled in centers with on-site CSS and those enrolled in centers without on-site CSS demonstrated virtually identical rates of left main artery and proximal LAD disease, with no significant differences in the rates of multivessel disease between the 2 groups (Table 2). The mean SYNTAX score was 22.13 in centers with and without on-site CSS $(P=.995)$. Furthermore, the number of patients in the high (>32), medium (23-32), and low $(\leq 22)$ SYNTAX categories was similar in the 2 groups (Table 2).

\section{Factors Associated With Referral for PCI Versus CABG}

Unadjusted analyses showed that a higher proportion of patients underwent PCI (rather than being referred for CABG) in centers without on-site CSS than in centers with on-site CSS $(65 \%$ vs $47 \% ; P<.001)$ (Figure $2, A$ ). Furthermore, in patients referred to CABG, mean SYNTAX score was significantly higher in centers without on-site CSS compared with those with on-site CSS (29 vs 26.7; $P=.018$ ) (Figure 2, B). Consistently, the proportion of patients in the high SYNTAX category $(>32)$ who were referred to PCI was twice as high in the centers without on-site CSS compared with those with on-site CSS (8\% vs $4 \%$ ). In addition to the differences in SYNTAX scores,
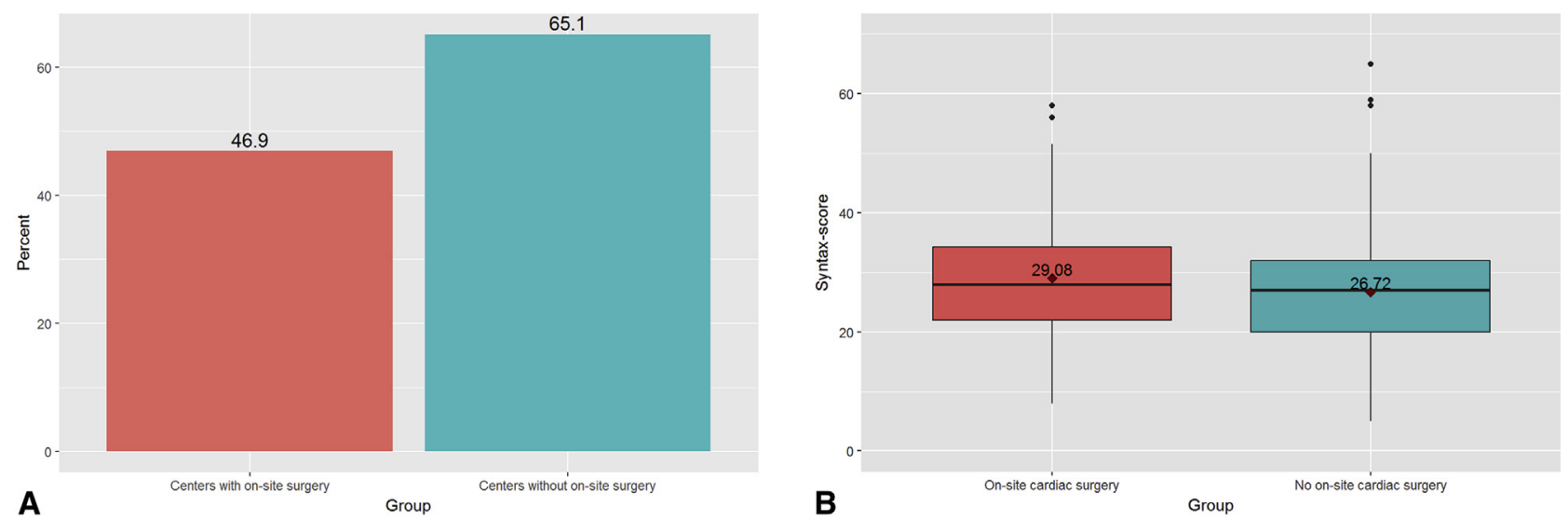

FIGURE 2. A, Bar graph of percentage of patients who underwent PCI by type of center. In centers without on-site cardiac surgery, $65.1 \%$ of patients underwent PCI, compared with $46.9 \%$ of patients from centers with on-site cardiac surgery $(P<.001)$. B, Boxplot of mean SYNTAX score of patients who were referred to the coronary artery bypass graft (CABG) SYNTAX score of patients referred for CABG from centers with and without on-site cardiac surgery (mean, $26.72 \pm 9.62$ and $29.08 \pm 9.75$, respectively; $P=.018$ ). 


\section{OR for PCI Procedure with $95 \% \mathrm{Cl}$}

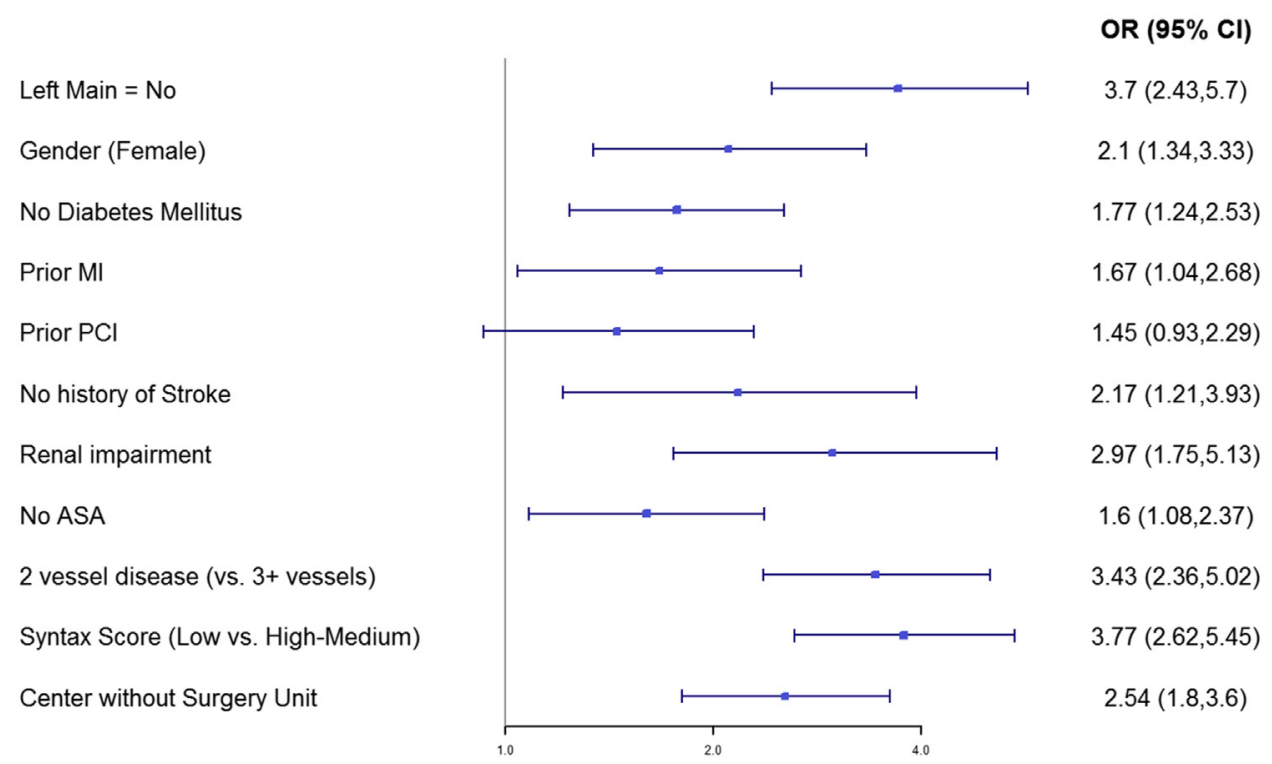

FIGURE 3. Logistic regression, predictors for referring patients for PCI. $O R$, Odds ratio; $P C I$, percutaneous coronary intervention; $C I$, confidence interval; $M I$, myocardial infarction; $A S A$, acetylsalicylic acid.

those who were referred to CABG from centers without CSS were older (mean age, 66 vs 64 years; $P=.048$ ). The mean Euroscore of patients referred to $\mathrm{CABG}$ was 1.23 , compared with 1.35 in those who underwent PCI $(P=.129)$. Among patients with a high SYNTAX score (>32) who underwent PCI, the mean Euroscore was 6.22 in centers with available on-site CSS, compared with 2.65 in centers without on-site CSS.

Consistent with these findings, multivariate logistic regression analysis showed that the absence of on-site CSS was an independent predictor for PCI versus CABG referral (Figure 3).

This analysis showed that patients enrolled in centers with only stand-alone interventional cardiology units were 2.5 times more likely to be referred to PCI compared with patients enrolled in centers with an available heart team and on-site CSS (odds ratio, 2.54; 95\% confidence interval [CI], 1.8-3.6). Additional independent predictors for PCI versus $C A B G$ included female sex, nondiabetic patients, renal impairment, absence of left main artery disease, 2-vessel versus 3-vessel disease and lower SYNTAX score (Figure 3). Similar results were obtained after further adjustment for center volume and Euroscore (Figures E2 and $\mathrm{E} 3$ ).

\section{Long-Term Survival Based on the Availability of On-Site CSS}

The 3-year cumulative survival probability was somewhat higher in patients admitted to centers with a heart team $(92.3 \%)$ compared with those without $(91 \%)$, but unadjusted analyses revealed no statistical significance (Figure 4, A). Nevertheless, comparison of outcomes by revascularization strategy showed that in centers with a heart team and on-site CSS, 3-year cumulative survival was significantly higher in patients treated with $\mathrm{CABG}$ compared with those treated with PCI (Figure 4, B). In contrast, in centers without on-site CSS, referral to CABG was not associated with improved survival (Figure 4,C).

Furthermore, results of a subgroup analysis of patients admitted to centers without on-site CSS demonstrated a lower 3-year cumulative survival probability in those with a medium to high SYNTAX score $(>22)$ compared with those with a lower SYNTAX score $(\leq 22)$ (Figure $5, A)$, whereas on-site CSS was associated with a higher 3-year cumulative survival probability in patients with a high-medium SYNTAX score $(>22)$, a rate not significantly different from the low SYNTAX $(\leq 22)$ group (Figure 5, $B$ ).

\section{Long-Term Survival in the Overall Study Cohort}

Unadjusted comparisons between the 2 revascularization strategies in the entire study cohort showed a long-term advantageous trend toward CABG (Figure 6), with a statistically significant treatment-by-time interaction effect. Thus, 9-month cumulative survival probabilities were similar in patients who underwent CABG and those who underwent PCI $(96.1 \%$ vs $96 \% ; P=.969)$, whereas landmark analysis showed that at the beginning of the ninth month following intervention, subsequent cumulative survival probabilities were significantly lower among those who underwent PCI $(P=.021)$ (Figure 6). Consistent with 

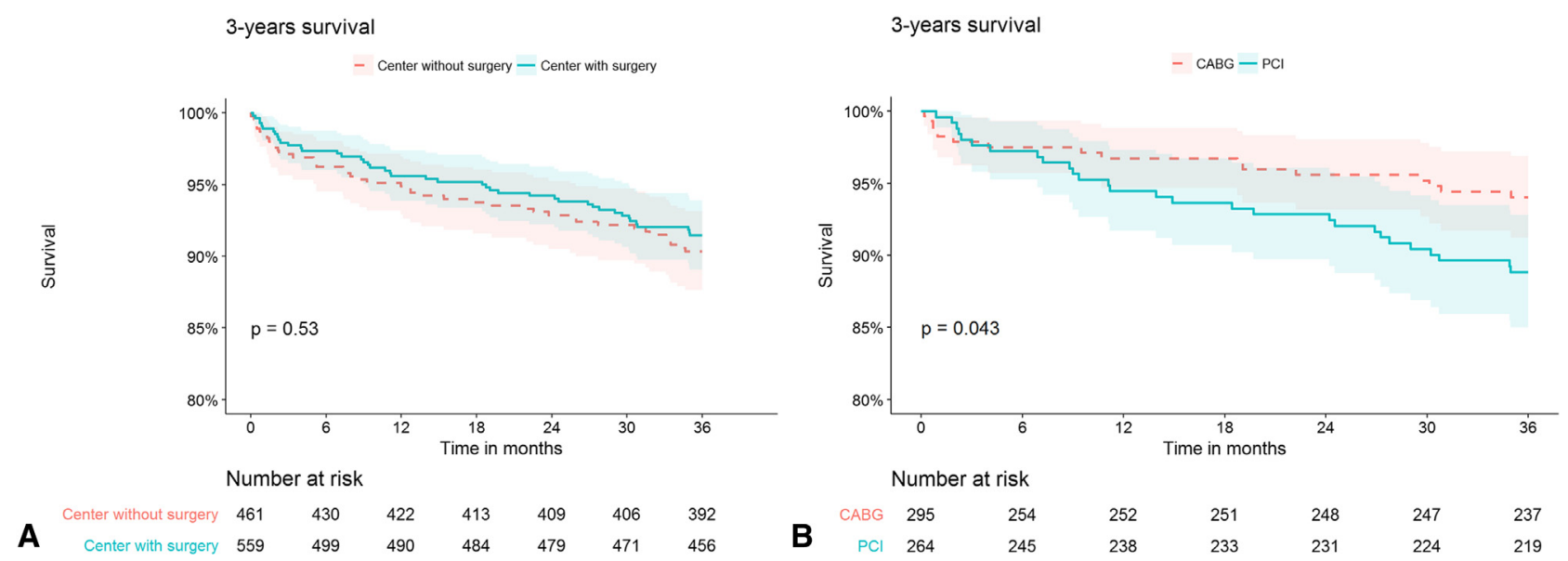

3-years survival

$-\mathrm{CABG}-\mathrm{PCI}$

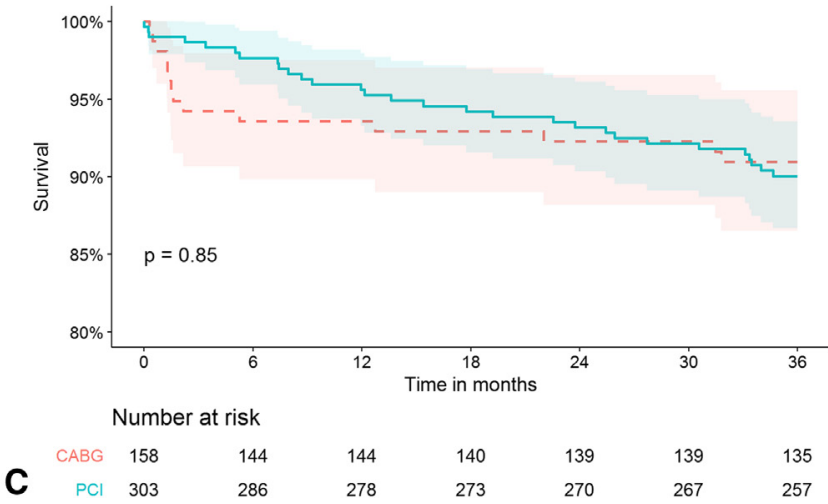

FIGURE 4. Three-year survival curves by centers with and without on-site cardiac surgery (A), by revascularization strategy in centers with on-site cardiac surgery (B), and by revascularization strategy in centers without on-site cardiac surgery (C). CABG, Coronary artery bypass graft; $P C I$, percutaneous coronary intervention.

these findings, multivariate analysis showed that referral to CABG in the entire MULTICAD cohort was independently associated with a significant $64 \%$ reduction in the risk of

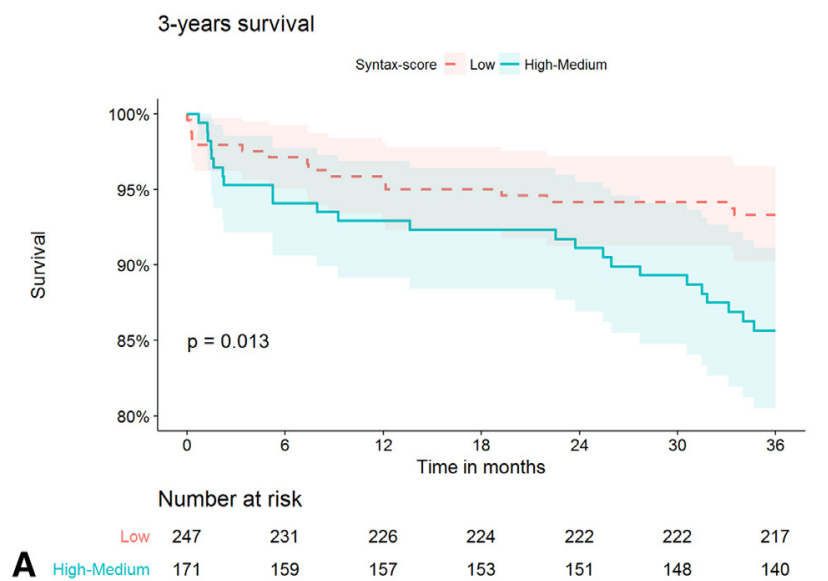

3-year mortality compared with referral to PCI $(P=.002)$. Because of an interaction with the time of revascularization strategy variable, we performed an

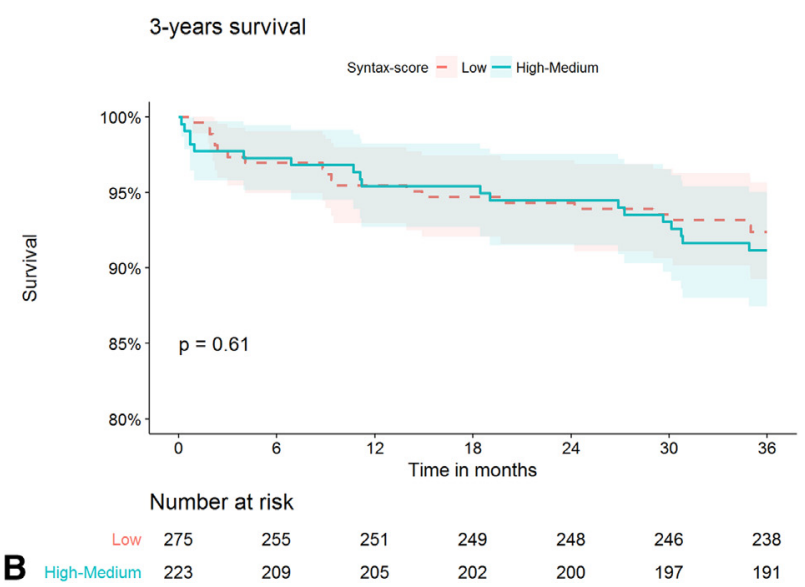

FIGURE 5. Three-year survival curves by SYNTAX score in centers without cardiac surgery services (A) and by SYNTAX score in centers with cardiac surgery services (B). Low SYNTAX, SYNTAX score $\leq 22$; high-medium SYNTAX, SYNTAX score $>22$. 


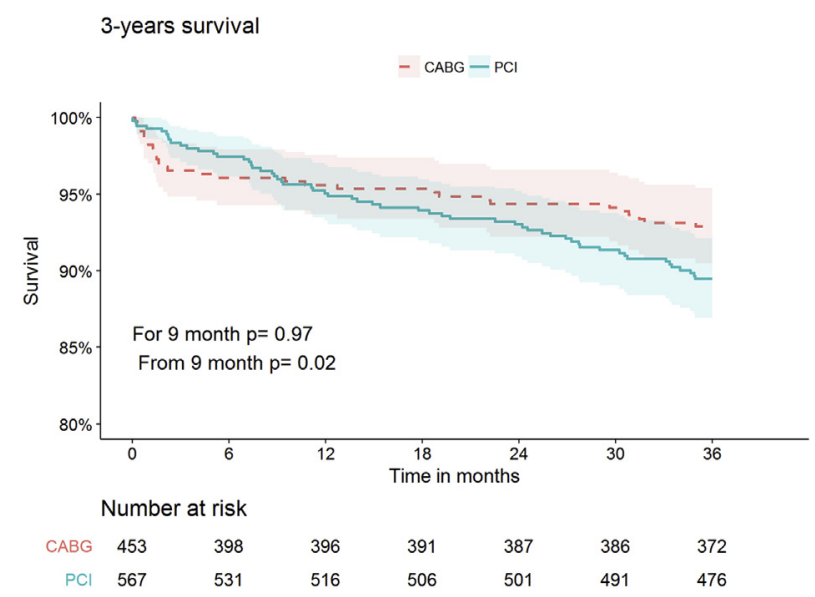

FIGURE 6. Overall 3-year survival curves by revascularization strategy. Kaplan-Meier for the whole period, with $P$ values for the first 9 months and from month 9 and thereafter. $C A B G$, Coronary artery bypass graft; $P C I$, percutaneous coronary intervention.

additional analysis by time, which showed that up to 9 months from admission, CABG was not associated with a survival advantage, but from 9 months on, CABG was associated with an $86 \%$ reduction in mortality risk compared with PCI $(P<.001)$. Additional predictors of 3 -year mortality included a SYNTAX score $>22$ $(P=.04)$, involvement of the left main coronary artery $(P=.03)$, age $>75$ years $(P=.001)$, atrial fibrillation $(P=.03)$, and diabetes mellitus $(P=.002)$ (Table 3$)$. Similar results were obtained after further adjustment for center volume and Euroscore (Tables E2-E5). A subanalysis among patients with available Euroscore parameters showed that the association between CABG and improved outcome was consistent from 9 months and thereafter (hazard ratio [HR], 0.27; 95\% CI, 0.09-0.86; $P=.027)$. A propensity score matching analysis also

TABLE 3. Adjusted COX model for 3-year mortality

\begin{tabular}{lcc}
\hline \multicolumn{1}{c}{ Variable } & HR $(\mathbf{9 5} \% \mathbf{C I})$ & $\boldsymbol{P}$ value \\
\hline Centers without surgery unit & $0.97(0.60-1.57)$ & .91 \\
SYNTAX high-medium vs low* & $1.80(1.04-3.12)$ & .04 \\
\hline Left main & $1.87(1.07-3.26)$ & .03 \\
Revascularization by CABG vs & $0.36(0.20-0.67)$ & .002 \\
$\quad$ PCI (all periods) & & \\
Revascularization by CABG vs & $0.99(0.42-2.38)$ & .99 \\
$\quad$ PCI (until 9 mo) & & \\
Revascularization by CABG vs & $0.14(0.06-0.36)$ & $<.001$ \\
$\quad$ PCI (starting from 9 mo) & & .75 \\
Male sex & $0.91(0.54-1.56)$ & .001 \\
Age $>75$ y & $2.43(1.47-4.01)$ & .03 \\
\hline Atrial fibrillation & $2.36(1.10-5.04)$ & .002 \\
\hline Diabetes mellitus & $2.25(1.37-3.68)$ & \\
\hline
\end{tabular}

$H R$, Hazard ratio; $C I$, confidence interval; $C A B G$, coronary artery bypass grafting; $P C I$, percutaneous coronary intervention. *High-medium, SYNTAX score $>22$; low, SYNTAX score $\leq 22$. yielded consistent results. Up to 9 months from admission, CABG was not associated with a significant survival advantage (HR, $1.3 ; \quad 95 \% \quad \mathrm{CI}, \quad 0.51-3.33$; $P=.58$ ), but from 9 months thereafter it was associated with a significant reduction in mortality risk compared with PCI (HR, 0.35; 95\% CI, 0.13-0.97; $P=.05$ ) (Table E6).

\section{DISCUSSION}

Our findings, derived from a real-life prospective cohort of patients enrolled in a national registry, provide several important implications regarding the effect of on-site CCS on management strategies and outcomes of patients with multivessel CAD. We have shown that (1) centers with stand-alone interventional cardiology units are significantly more likely to refer patients with multivessel CAD to PCI compared with centers with an available heart team and on-site CSS; (2) the SYNTAX threshold for CABG referral in centers without on-site CSS is significantly higher compared with the respective threshold in centers with on-site CSS; and (3) in centers with available on-site CSS, CABG is associated with improved 3-year survival compared with PCI.

Appropriate patient selection via a multidisciplinary approach is the key for successful patient management by either PCI or CABG. Our findings further emphasize the clinical importance of a heart team approach comprising a cardiac surgeon, an interventional cardiologist, and a general cardiologist, which should be mandatory even in centers with stand-alone interventional cardiology units, where the heart team should meet in either a live or digital weekly or fortnightly session to present and comprehensively discuss each patients with multivessel CAD.

With ischemic heart disease on the rise worldwide, an increasing number of attending heart team physicians in stand-alone interventional cardiology units focus less on patient-oriented decision-making regarding the preferable strategy for coronary revascularization, CABG and/or PCI. In centers lacking on-site CSS, the vast majority of virtually all patients with multivessel disease who undergo PCI do not benefit from a heart team discussion before their procedure. Although differences in patient characteristics may explain some of the variables in revascularization decision making, much of the variance is physiciandriven, resulting in potentially higher rates of PCI.

In certain circumstances, such as an emergency, performing PCI immediately after the diagnostic coronary angiogram is certainly appropriate and has been established as safe and effective compared with delayed PCI. However, this approach is questionable in more stable clinical situations and in patients with complex CAD. Hasty therapeutic decision making may occur more frequently in centers without on-site CSS, when a surgical consultant is not immediately available. 
The rate of isolated CABG has declined over the last decade. ${ }^{9}$ In clinical practice, physicians tend to underuse surgical coronary revascularization in patients with CAD who are considered appropriate candidates. Hemingway and colleagues ${ }^{10}$ reported that $26 \%$ of patients with appropriate indications for CABG were eventually treated medically, an approach that resulted in adverse clinical outcomes.

Our study demonstrates how the lack of a heart team in real-life practice tends to rely less on patient-oriented decision making when managing patients with multivessel CAD (including the LAD artery or left main coronary artery), referring them to PCI rather than CABG despite indications for surgical revascularization based on current guidelines. ${ }^{6}$ Because all our patients had an American College of Cardiology/American Heart Association class I indication for $\mathrm{CABG}$, we believe that our results demonstrate diminished guideline adherence in centers lacking on-site CSS. Although our study focused on patients recruited from January through April 2013, nothing has changed in terms of standard practice in centers lacking on-site CSS since then.

Moreover, patients referred to surgical revascularization in stand-alone interventional cardiology units lacking a heart team were older and at higher surgical risk compared with those referred to CABG from centers with CSS and a heart team. Furthermore, the anatomy of the coronary arteries was more complex in those referred to CABG from centers without a heart team. Although the mean SYNTAX score was similar in centers with and those without on-site CSS, patients referred to CABG from hospitals without on-site CSS had significantly higher mean SYNTAX scores. Previous studies have identified SYNTAX score as an important determinant of PCI outcomes. ${ }^{11,12}$ In our results, a higher SYNTAX score was an independent predictor for mortality in all patients who underwent revascularization due to complex CAD. We found that unlike in hospitals with on-site CSS, patients with a higher SYNTAX score experienced significantly higher mortality rates than those with a low SYNTAX score in hospitals without on-site CSS. However, in centers with available on-site CSS, mortality was similar in patients in the low and high SYNTAX groups. The obvious explanation is that in the absence of a heart team, more complex CAD cases are treated differently. Some patients are treated by PCI instead of CABG, and others experience delayed surgical intervention, resulting in less favorable outcomes. The late 3-year follow-up in our study included mortality as the only outcome. We speculate that had other outcomes (eg, major adverse cardiac events) been evaluated, CABG would have demonstrated additional advantages, given that in non-cardiac surgery centers patients with higher SYNTAX scores would have undergone PCI.
Multivariate logistic regression analysis identified the absence of on-site CSS and a heart team as an independent predictor for referring a patient with multivessel CAD to PCI rather than to CABG. This analysis supports the hypothesis that stand-alone interventional cardiology units reach different clinical decisions for revascularization strategies compared with interventional cardiology units in centers with on-site CSS. Moreover, the existence of on-site CSS becomes a significant factor when choosing a revascularization techniques, along with involvement of the left main coronary artery, diabetes mellitus, previous stroke, and SYNTAX score.

At a 3-year follow-up, mortality in patients who underwent $\mathrm{CABG}$ was higher in those referred from centers without on-site CSS than in those referred from centers with on-site CSS and a heart team, even though the different was not statistically significant. Notably, CABG was associated with significantly lower 3-year mortality, especially in centers with available on-site CSS, suggesting that a heart team approach stratifies patients with multivessel disease to the appropriate surgical intervention.

\section{CONCLUSIONS AND CLINICAL IMPLICATIONS}

Lack of a heart team increases the potential for significant bias in referral patterns for coronary revascularization in stand-alone interventional cardiology units. Implementation of a heart team in routine cardiac care has great potential for positively influencing the quality of cardiovascular care in patients with multivessel CAD who are referred to revascularization. A heart team approach should be mandatory even in centers without on-site CSS. Furthermore, digital communication between interventional cardiologists and cardiac surgeons in centers lacking on-site CCS needs to be improved to achieve consensus on management strategies.

\section{Limitations}

Our findings on the difference in mortality between centers with and without on-site CSS did not reach statistical significance. Although these findings were consistent overall and across subgroups, the number of events was limited and thus perhaps the study lacked power. A larger cohort and/or a longer follow-up period could yield more statistically significant results supporting lower mortality in patients with complex CAD referred for surgical revascularization based on a heart team consensus compared with patients referred to surgery without a team based approach. Although the differences between patients who underwent PCI and those who underwent $\mathrm{CABG}$ are large, owing to a lack of consistent data collection of all STS scores or Euroscore parameters of all study patients (available for only $57 \%$ of the cohort), we could not calculate the predicted patient risk according to STS score or Euroscore, and thus could not determine the superiority 
of real-life revascularization strategy. A formal heart team was coordinated according to the discretion of the treating physician, rather than relying on uniform decision making. Therefore, we could not assess the effect of an on-site cardiac surgery heart team approach on patient outcomes.

\section{Webcast}

You can watch a Webcast of this AATS meeting presentation by going to: https://aats.blob.core.windows.net/ media/17AM/2017-05-01/RM311/05-01-17_Room311_ 1630_Raanani.mp4.

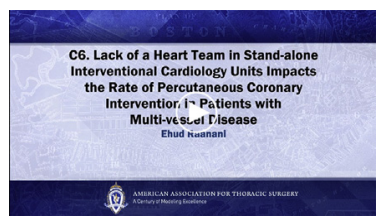

\section{Conflict of Interest Statement}

Authors have nothing to disclose with regard to commercial support.

\section{References}

1. McAloon CJ, Boylan LM, Hamborg T, Stallard N, Osman F, Lim PB, et al. The changing face of cardiovascular disease 2000-2012: an analysis of the World Health Organization Global Health Estimates data. Int J Cardiol. 2016;224: 256-64.

2. Serruys PW, Morice MC, Kappetein AP, Colombo A, Holmes DR, Mack MJ, et al. Percutaneous coronary intervention versus coronary-artery bypass grafting for severe coronary artery disease. $N$ Engl J Med. 2009;360:961-72.

3. Influence of diabetes on 5-year mortality and morbidity in a randomized trial comparing CABG and PTCA in patients with multi-vessel disease: the Bypass
Angioplasty Revascularization Investigation (BARI). Circulation. 1997;96: 1761-9.

4. Farkouh ME, Domanski M, Sleeper LA, Siami FS, Dangas G, Mack M, et al. Strategies for multi-vessel revascularization in patients with diabetes. $N$ Engl J Med. 2012;367:2375-84.

5. Cho Y, Shimura S, Aki A, Furuya H, Okada K, Ueda T. The SYNTAX score is correlated with long-term outcomes of coronary artery bypass grafting for complex coronary artery lesions. Interact Cardiovasc Thorac Surg. 2016;23: 125-32.

6. Kolh P, Windecker S, Alfonso F, Collet JP, Cremer J, Falk V, et al. 2014 ESC/EACTS Guidelines on myocardial revascularization: the Task Force on Myocardial Revascularization of the European Society of Cardiology (ESC) and the European Association for Cardio-Thoracic Surgery (EACTS). Developed with the special contribution of the European Association of Percutaneous Cardiovascular Interventions (EAPCI). Eur J Cardiothorac Surg. 2014;46: 517-92.

7. Zheng Z, Rao C, Du J. Is the era of the heart team coming? J Thorac Cardiovasc Surg. 2015;150:1664-5.

8. Kolh P, Kurlansky P, Cremer J, Lawton J, Siepe M, Fremes S. Transatlantic editorial: a comparison between European and North American guidelines on myocardial revascularization. J Thorac Cardiovasc Surg. 2016;152:304-16.

9. Kim LK, Looser P, Swaminathan RV, Minutello RM, Wong SC, Girardi L, et al. Outcomes in patients undergoing coronary artery bypass graft surgery in the United States based on hospital volume, 2007 to 2011. J Thorac Cardiovasc Surg. 2016;151:1686-92.

10. Hemingway H, Crook AM, Feder G, Banerjee S, Dawson JR, Magee P, et al Underuse of coronary revascularization procedures in patients considered appropriate candidates for revascularization. N Engl J Med. 2001;344:645-54.

11. Valgimigli M, Serruys PW, Tsuchida K, Vaina S, Morel MA, van den Brand MJ et al. Cyphering the complexity of coronary artery disease using the syntax score to predict clinical outcome in patients with three-vessel lumen obstruction undergoing percutaneous coronary intervention. Am J Cardiol. 2007;99:1072-81.

12. Garg S, Serruys PW, Silber S, Wykrzykowska J, van Geuns RJ, Richardt G, et al. The prognostic utility of the SYNTAX score on 1-year outcomes after revascularization with zotarolimus- and everolimus-eluting stents: a substudy of the RESOLUTE All Comers Trial. JACC Cardiovasc Interv. 2011;4:432-41.

Key Words: adult, coronary artery bypass grafts, coronary stents, percutaneous coronary intervention 


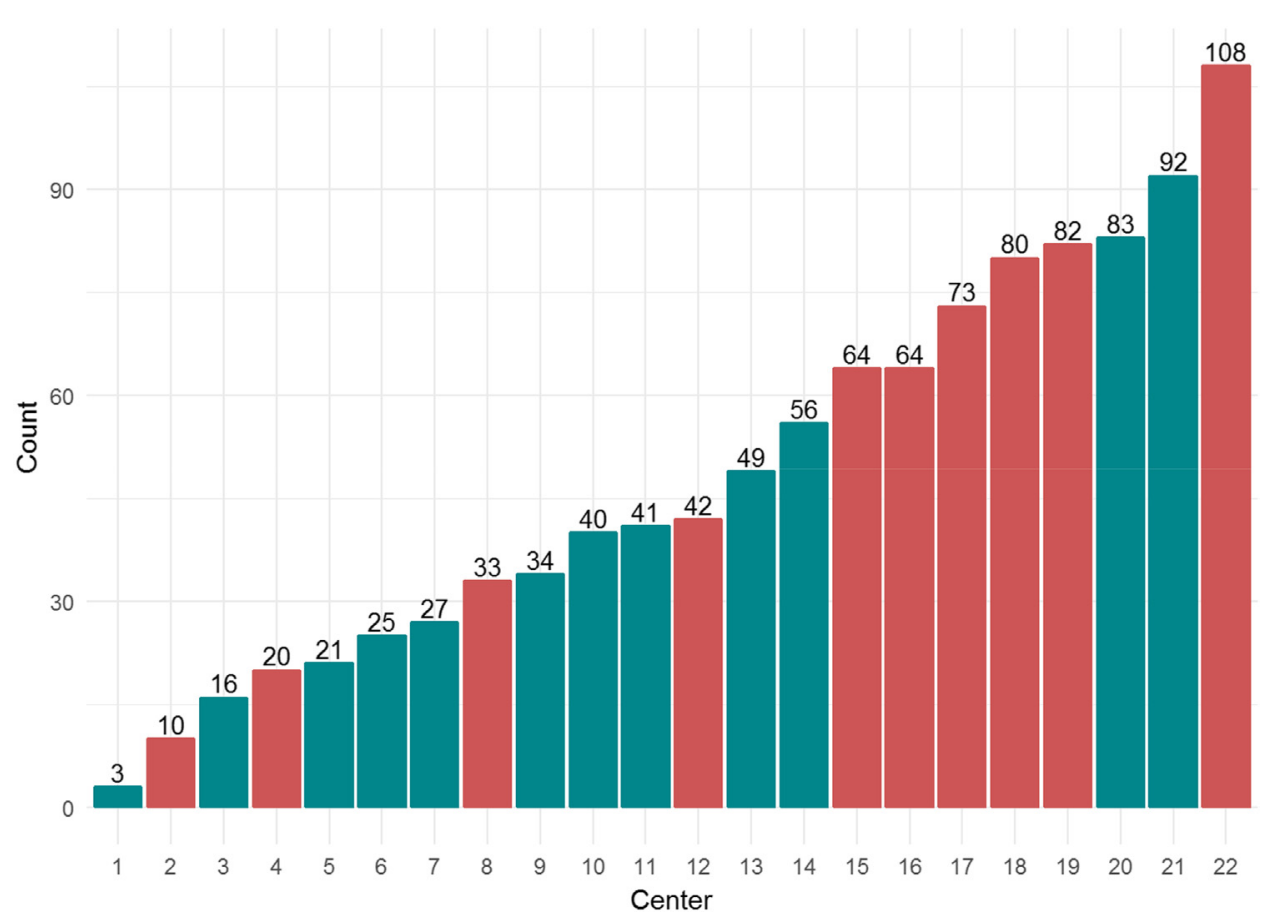

FIGURE E1. Bar plot of patient distribution by center. Red bars indicate centers with on-site cardiac surgery unit (CSS); blue bars, centers without on-site CSS.

\section{OR for PCI Procedure with $95 \% \mathrm{CI}$}

Left Main = No
Gender (Female)
No Diabetes Mellitus
Prior MI
Prior PCl
No history of Stroke
Renal impairment
No ASA
2 vessel disease (vs. 3+ vessels)
Syntax Score (Low vs. High-Medium)
Center without Surgery Unit

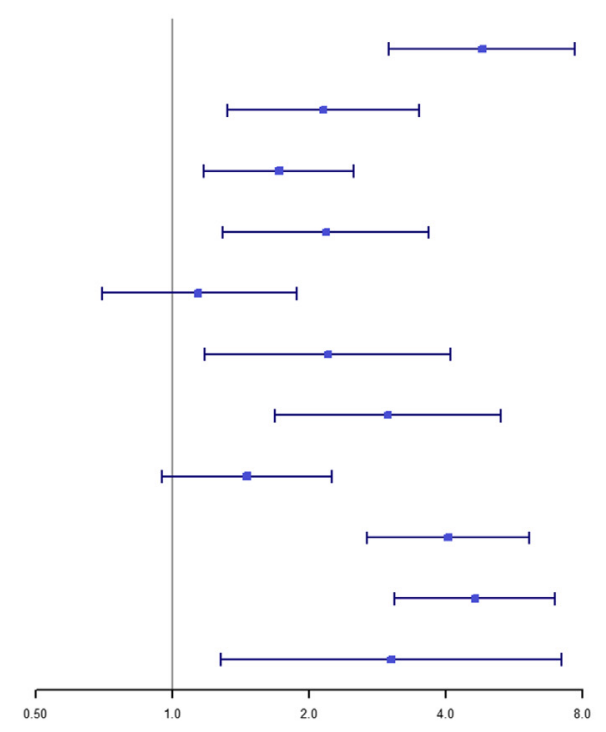

OR $(95 \% \mathrm{Cl})$

$4.81(3,7.7)$

$2.15(1.32,3.5)$

$1.72(1.17,2.51)$

$2.18(1.29,3.67)$

$1.14(0.7,1.88)$

$2.2(1.18,4.1)$

$2.98(1.68,5.29)$

$1.46(0.95,2.24)$

$4.05(2.68,6.12)$

$4.64(3.09,6.97)$

$3.03(1.28,7.19)$

Center as a random effect has standard deviation of 0.8702 .

FIGURE E2. Mixed-effects logistic regression with the center variable adjusted as a random variable. OR, Odds ratio; $P C I$, percutaneous coronary intervention; $C I$, confidence interval; $M I$, myocardial infarction; $A S A$, acetylsalicylic acid. 


\section{OR for PCI Procedure with $95 \% \mathrm{CI}$}

Left Main = No
Gender (Female)
No Diabetes Mellitus
Prior Ml
Prior PCl
No history of Stroke
Renal impairment
No ASA
2 vessel disease (vs. 3+ vessels)
Syntax Score (Low vs. High-Medium)
EuroSCORE (per point)
Center without Surgery Unit

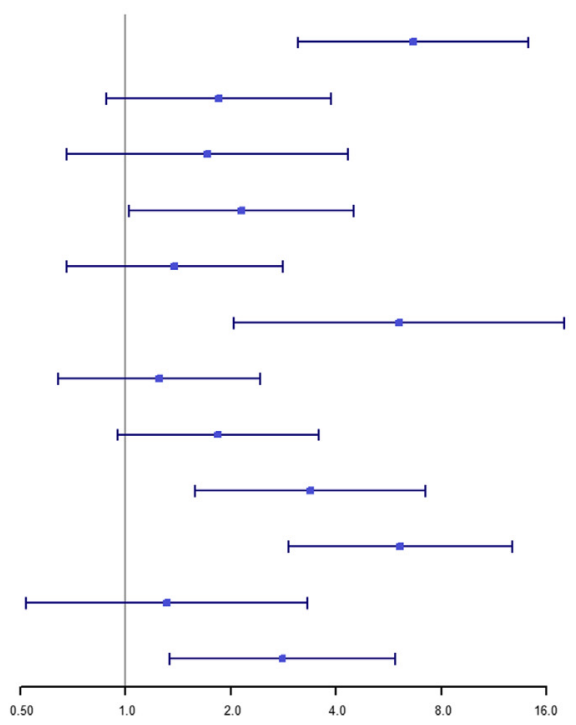

OR $(95 \% \mathrm{Cl})$

$6.67(3.12,14.25)$

$1.85(0.88,3.87)$

$1.71(0.68,4.32)$

$2.14(1.02,4.49)$

$1.38(0.68,2.82)$

$6.06(2.04,18)$

$1.25(0.64,2.43)$

$1.84(0.95,3.58)$

$3.38(1.58,7.22)$

$6.11(2.92,12.79)$

$1.31(0.52,3.32)$

$2.81(1.34,5.9)$

Center as a random effect has standard deviation of 0.78 .

FIGURE E3. Mixed-effects logistic regression with the center variable adjusted as a random variable including Euroscore. OR, Odds ratio; $P C I$, percutaneous coronary intervention; $C I$, confidence interval; $M I$, myocardial infarction; ASA, acetylsalicylic acid.

TABLE E1. Complications and outcomes at 30 days

\begin{tabular}{lccr}
\hline Complication/outcome & $\begin{array}{c}\text { Center } \\
\text { without CSS, } \\
\mathbf{n}(\%)\end{array}$ & $\begin{array}{c}\text { Center } \\
\text { with CSS, } \\
\text { n (\%) }\end{array}$ & $\begin{array}{c}\boldsymbol{P} \\
\text { value }\end{array}$ \\
\hline Mortality & $6(1.3)$ & $6(1.1)$ & 1.00 \\
\hline Unstable angina pectoris & $4(1)$ & $2(0.4)$ & .49 \\
\hline Myocardial infarction & $3(0.7)$ & $3(0.6)$ & 1.00 \\
Congestive heart failure & $1(0.2)$ & $3(0.6)$ & .78 \\
\hline Cardiogenic shock & $2(0.5)$ & $5(1)$ & .62 \\
\hline Free wall rapture & $1(0.2)$ & $1(0.2)$ & 1.00 \\
\hline Ventricular fibrillation & $3(0.7)$ & $2(0.4)$ & .83 \\
\hline Acute VSD & $1(0.2)$ & $0(0)$ & .92 \\
\hline Pericarditis & $0(0)$ & $2(0.4)$ & .58 \\
\hline Ischemic stroke & $1(0.2)$ & $1(0.2)$ & 1.00 \\
\hline Hemorrhagic stroke & $1(0.2)$ & $1(0.2)$ & 1.00 \\
\hline Acute renal failure & $9(2.1)$ & $20(3.8)$ & .18 \\
\hline Sepsis & $4(0.9)$ & $7(1.3)$ & .78 \\
\hline
\end{tabular}

CSS, Cardiac surgery services; VSD, ventricular septal defect.
TABLE E2. Mixed-effects Cox model by center volume for mortality over 3 years

\begin{tabular}{lccc}
\hline \multicolumn{1}{c}{ Variable } & HR & $\mathbf{2 . 5} \%$ & $\mathbf{9 7 . 5} \%$ \\
\hline Centers without cardiac surgery unit & 0.98 & 0.57 & 1.66 \\
\hline SYNTAX high-medium vs low* & 1.82 & 1.05 & 3.15 \\
\hline Left main & 1.91 & 1.09 & 3.34 \\
Revascularization by PCI vs CABG & 2.86 & 1.52 & 5.36 \\
Male sex & 0.91 & 0.53 & 1.56 \\
Age $>$ 75 y & 2.36 & 1.43 & 3.91 \\
Atrial fibrillation & 2.41 & 1.12 & 5.21 \\
Diabetes mellitus & 2.27 & 1.38 & 3.73 \\
\hline Cen
\end{tabular}

Center as a random effect has standard deviation of 0.22 . $H R$, Hazard ratio; $P C I$, percutaneous coronary intervention; $C A B G$, coronary artery bypass grafting. *High-medium: SYNTAX score $>22$; low: SYNTAX score $\leq 22$. 
TABLE E3. Mixed-effects Cox model by center volume starting from 9 months to mortality at 3 years

\begin{tabular}{lccr}
\hline \multicolumn{1}{c}{ Variable } & HR & $\mathbf{2 . 5} \%$ & $\mathbf{9 7 . 5} \%$ \\
\hline Centers without cardiac surgery unit & 0.68 & 0.35 & 1.33 \\
SYNTAX high-medium vs low* & 3.25 & 1.52 & 6.95 \\
\hline Left main & 1.98 & 0.95 & 4.15 \\
Revascularization by PCI vs CABG & 7.00 & 2.77 & 17.70 \\
Male sex & 0.86 & 0.41 & 1.80 \\
Age $>75$ y & 1.91 & 0.94 & 3.88 \\
Atrial fibrillation & 3.13 & 1.05 & 9.29 \\
Diabetes mellitus & 1.87 & 0.96 & 3.63 \\
\hline
\end{tabular}

Center as a random effect has standard deviation of $0.02 . H R$, Hazard ratio; $P C I$, percutaneous coronary intervention; $C A B G$, coronary artery bypass grafting. *High-medium: SYNTAX score $>22$; low: SYNTAX score $\leq 22$
TABLE E5. Mixed-effects Cox model by center volume with Euroscore from 9 months to mortality at 3 years

\begin{tabular}{lccr}
\hline \multicolumn{1}{c}{ Variable } & HR & $\mathbf{2 . 5} \%$ & $\mathbf{9 7 . 5} \%$ \\
\hline Centers without surgery unit & 0.76 & 0.31 & 1.86 \\
SYNTAX high-medium vs low* & 1.79 & 0.63 & 5.04 \\
\hline Left main & 4.86 & 1.81 & 13.02 \\
Revascularization by PCI vs CABG & 3.63 & 1.16 & 11.35 \\
Euroscore & 1.31 & 1.11 & 1.54 \\
Atrial fibrillation & 2.01 & 0.52 & 7.77 \\
Diabetes mellitus & 1.95 & 0.83 & 4.60 \\
\hline
\end{tabular}

Center as a random effect has standard deviation of 0.02 . $H R$, Hazard ratio; $P C I$, percutaneous coronary intervention; $C A B G$, coronary artery bypass grafting. *High-medium, SYNTAX score $>22$; low, SYNTAX score $\leq 22$.
TABLE E4. Mixed-effects Cox model by center volume with Euroscore for mortality at 3 years

\begin{tabular}{lccc}
\hline \multicolumn{1}{c}{ Variable } & HR & $\mathbf{2 . 5} \%$ & $\mathbf{9 7 . 5} \%$ \\
\hline Centers without surgery unit & 1.22 & 0.67 & 2.22 \\
SYNTAX high-medium vs low* & 1.26 & 0.65 & 2.47 \\
Left main & 3.05 & 1.59 & 5.85 \\
Revascularization by PCI vs CABG & 1.64 & 0.78 & 3.46 \\
Euroscore & 1.28 & 1.17 & 1.41 \\
Atrial fibrillation & 1.97 & 0.82 & 4.75 \\
Diabetes mellitus & 2.60 & 1.44 & 4.71 \\
\hline
\end{tabular}

Center as a random effect has standard deviation of 0.02 . $H R$, Hazard ratio; $P C I$, percutaneous coronary intervention; $C A B G$, coronary artery bypass grafting. *High-medium: SYNTAX score $>22$; low: SYNTAX score $\leq 22$.
TABLE E6. Propensity score matching by revascularization strategy (PCI vs CABG): mortality over 3 years

\begin{tabular}{|c|c|c|}
\hline Revascularization by PCI vs CABG & HR $(95 \%$ CI $)$ & $P$ value \\
\hline Until 9 mo & $0.77(0.30-1.95)$ & .58 \\
\hline 9 mo and thereafter & $2.85(1.03-7.92)$ & .05 \\
\hline \multicolumn{3}{|c|}{$\begin{array}{l}\text { Matching was done for the following variables: left main disease, sex, diabetes } \\
\text { mellitus, previous myocardial infarction, previous PCI, history of stroke, renal } \\
\text { impairment, } 3 \text { or more CADs (vs } 2 \text { CADs), SYNTAX score }>32 \text { versus } \leq 32 \text {, and cen- } \\
\text { ters with on-site cardiac surgery services. For a } 1: 1 \text { match, } 179 \text { patients were treated } \\
\text { with PCI and } 179 \text { were treated with CABG. PCI, Percutaneous coronary intervention } \\
C A B G \text {, coronary artery bypass grafting; } H R \text {, hazard ratio; } C I \text {, confidence interval. }\end{array}$} \\
\hline
\end{tabular}

